\title{
Viral Attachment Induces Rapid Recruitment of an Innate Immune Sensor (TRIM5a) to the Plasma Membrane
}

\author{
Seiga Ohmine ${ }^{a}$ Raman Deep Singh ${ }^{b}$ David L. Marks ${ }^{b}$ Melissa A. Meyer ${ }^{a}$ \\ Richard E. Pagano ${ }^{b}$ Yasuhiro lkeda ${ }^{a}$ \\ Departments of a Molecular Medicine and ${ }^{b}$ Biochemistry and Molecular Biology, Mayo Clinic, Rochester, Minn., USA
}

\section{Key Words}

HIV · Innate immunity · Membrane rafts · Plasma

membrane $\cdot$ Restriction factor $\cdot$ TRIM5a

\begin{abstract}
TRIM5a (tripartite motif 5a) acts as a pattern recognition receptor specific for the retrovirus capsid lattice and blocks infection by HIV-1 immediately after entry. However, the precise mechanisms underlying this rapid recognition of viral components remain elusive. Here, we analyzed the influence of viral exposure on TRIM5a. Total internal reflection fluorescence microscopy and lipid flotation assays revealed rapid recruitment of a TRIM5a subpopulation to the plasma membrane (PM) upon exposure to vesicular stomatitis virus-G-pseudotyped HIV-1 viral-like particles (VLPs), but not to envelope (Env)-less HIV-1 VLPs. TRIM5a signals were frequently colocalized with those of HIV-1 capsid at the PM. Exposure to HIV-1 Env-pseudotyped HIV-1 vectors also triggered translocation of endogenous TRIM5a to lipid microdomains within human T cells. Similarly, clustering of lipid microdomains by a glycosphingolipid stereoisomer resulted in rapid TRIM5a recruitment to the PM. Of note, recruitment of endogenous rhesus TRIM5a to the PM prior to HIV1 infection significantly increased the potency of viral re-
\end{abstract}

striction. Our data therefore suggest the importance of TRIM5a recruitment to the PM for TRIM5a-mediated innate immune sensing and restriction of retroviral infection.

Copyright $\odot 2013$ S. Karger AG, Basel

\section{Introduction}

TRIM5 $a$ (tripartite motif $5 a$ ) is one of the first identified host cell proteins which plays a key role in speciesspecific retroviral tropism [1-4]. TRIM proteins are characterized by the sequential N-terminal presentation of domains: RING, B-box 2 and coiled coil $[5,6]$, while the $5 a$ isoform contains a C-terminal B30.2 (PRYSPRY) domain and variations in this domain dictate the potency and specificity of the restriction against particular retroviruses [7-10]. The viral determinants of susceptibility to restriction are mapped to the capsid proteins [11-14]. Studies have demonstrated that the rhesus monkey TRIM5 $\alpha$ (TRIM5 $\alpha$ rh) rapidly detects the mature core of HIV-1 at a postentry, pre-integration stage in the viral life cycle $[3,4,15,16]$. Recognition by TRIM $5 \alpha$ results in premature disassembly or accelerated degradation of the viral core, leading to defective viral reverse transcription $[9,17]$.

\section{KARGER}

E-Mail karger@karger.com

www.karger.com/jin
(C) 2013 S. Karger AG, Basel

1662-811X/13/0054-0414\$38.00/0
Dr. Yasuhiro Ikeda

Department of Molecular Medicine, Mayo Clinic

Guggenheim 18-11c, 200 First Street SW

Rochester, MN 55905 (USA)

E-Mail ikeda.yasuhiro@mayo.edu 
Germline-encoded intracellular sensors recognize incoming pathogens through their specific molecular patterns as foreign entities [18, 19]. RIG-I and MDA5 rapidly recognize viral components and promote an antiviral state within the cell [20-22]. Similarly, the TRIM5 protein recognizes the retroviral capsid lattice, which stimulates the TAK1 kinase complex and activates downstream innate immune signaling pathways [23]. Although these intracellular viral sensors play critical roles in innate immunity, where and how they 'sense' the molecular signatures of specific pathogens remain largely unknown.

The host cell plasma membrane (PM) serves as the first physical barrier against viral entry, whereas viruses often target PM lipid microdomains as stable platforms to initiate infection. These membrane raft domains, which are enriched in cholesterol and sphingolipids [24], have been implicated in many cellular processes such as signal transduction and endocytosis, but have also been appreciated to play a role in HIV-1 entry. Cellular receptors and coreceptors for the virus reside in membrane raft fractions [25-28] and cholesterol promotes the clustering of CD4, as well as coreceptors CXCR4 and CCR5, on the PM for efficient binding and absorption of incoming virions [26, $29,30]$. Pharmacological disruption of target cell membrane rafts prevents virion entry [26, 27, 31-33], implicating these membrane raft domains as HIV-1 entry hot spots.

Recently, we and others have demonstrated that TRIM5 $\alpha$ localizes in lipid microdomains $[34,35]$, while cholesterol depletion through $\beta$-cyclodextrin, which primarily acts on PM-associated cholesterol, impairs the TRIM5 $\alpha$-mediated retroviral restriction [35]. Additionally, we have identified increased levels of endogenous human TRIM5 $\alpha$ in PM lipid microdomains after shortterm stimulation with a synthetic glycosphingolipid ana$\log$ [36]. Based on these observations, we hypothesized that a subset of TRIM5arh can be recruited to the PM for viral capsid recognition. To test this hypothesis, we examined the possible TRIM5arh localization at the PM upon viral exposure. Our data demonstrated that TRIM5arh was rapidly recruited to the inner leaflet of the host cell PM upon HIV-1 virus-like particle (VLP) binding. Total internal reflection fluorescence (TIRF) microscopy analysis identified frequent colocalization events of TRIM5arh and HIV-1 VLP signals. Moreover, the aggregation of PM lipid microdomains by the incorporation of a synthetic glycosphingolipid also induced rapid PM recruitment of TRIM5 $\mathrm{arh}$. The prerecruitment of TRIM5arh to the PM increased the TRIM5arh- mediated restriction of HIV-1 infection. Our findings therefore underscore the importance of rapid TRIM $5 a$ recruitment to the PM lipid microdomains for innate TRIM5arh-mediated sensing and restriction of retroviral infection.

\section{Materials and Methods}

\section{Cell Culture}

All cells were maintained in Dulbecco's modified Eagle's medium (DMEM) supplemented with 10\% fetal bovine serum (FBS) and antibiotics. Serum starvation conditions were maintained with $0.5 \%$ serum-supplemented DMEM. FRhK4 cells stably expressing TRIM5arh with C-terminally labeled mCherry (FRhK4T5aCh), C-terminally HA-tagged TRIM5arh (FRhK4T5a) and FRhK4 cells stably knocked down for TRIM5arh have been described previously $[35,37]$.

\section{Fluorescence and TIRF Microscopy}

Cells were grown on acid-washed and sterilized high-performance cover glasses (Zeiss, Thornwood, N.Y., USA). When cells were ready for imaging, cover glasses were washed in phosphatebuffered saline (PBS), fixed in 4\% paraformaldehyde PBS solution and then washed in molecular-grade water. Cover glasses were mounted on standard microscope slides using Slow Fade Gold antifade reagent (Invitrogen, Grand Island, N.Y., USA). Fluorescence microscopy was performed using a fluorescence microscope (IX70; Olympus, Tokyo, Japan) equipped with $\times 601.4$ NA or $\times 100$ 1.35 NA oil immersion objectives. Images were acquired using a QuantEM:512SC (Photometrics) CCD camera. For quantitation, all photomicrographs in a given experiment were exposed and processed identically for a given fluorophore and were analyzed using the MetaMorph image processing program (version 7.3.2; Universal Imaging). Images were prepared for individual figures using Photoshop CS (Adobe, San Jose, Calif., USA). TIRF microscopy was carried out using an Olympus attachment for the IX70 microscope. No deconvolution, 3D reconstructions, surface or volume rendering, or $\gamma$ adjustments were performed.

\section{Generation of HIV-1 VLPS}

HIV-1 green fluorescent protein (GFP) VLPs were prepared with a codon-optimized HIV-1 CA plasmid [37] where the p24 sequence was fused with GFP, pCMV-R8.91 and pMD.G [38] by transfection with FuGene 6 (Roche) in 293T cells. GFP vectors were prepared with a GFP-expressing pSIN-CSGWdINotI [37], pCMV-R8.91 and pMD.G [38] using FuGene 6 in 293T cells. GFPcarrying simian immunodeficiency virus (SIV) vectors were prepared using FuGene 6 as described previously [39, 40]. For HIV-1 envelope (Env)-pseudotyped particles, pDOL HIV1-Env [41] was used in place of pMD.G. SIVmac-based vector plasmids, pSIV3+ and pSIV-GFP, were kindly provided by Dr. F.L. Cossett [42]. The vector-containing culture supernatant was collected and filtered through a $0.45-\mu \mathrm{m}$ syringe filter $48-72 \mathrm{~h}$ after transfection. VLPs or virus vectors were centrifuged at $70,000 \mathrm{~g}$ for $2 \mathrm{~h}$ at $4^{\circ} \mathrm{C}$, washed and resuspended in PBS. Bovine serum albumin (BSA)-coated nanoparticles were used at a concentration of $0.12 \mathrm{nM}$ and have been described previously [43]. 
Detergent-Free Separation of Higher Buoyant Density Fractions The following procedure is based on that of Macdonald and Pike [44]. All procedures were performed on ice. FRhK4 cells in 150-mm plates (4 plates/treatment) were washed with PBS and then scraped into base buffer ( $20 \mathrm{mM}$ Tris- $\mathrm{HCl}, \mathrm{pH} 7.8 ; 250 \mathrm{mM}$ sucrose; $1 \mathrm{mM}$ $\mathrm{CaCl}_{2}$ and $1 \mathrm{mM} \mathrm{MgCl}_{2}$ ) supplemented with protease inhibitors (Sigma, St. Louis, Mo., USA). Cells were mechanically lysed by passage through a 22 -gauge 1.5 -inch needle 60 times. Lysates were centrifuged at $1,000 \mathrm{~g}$ for $10 \mathrm{~min}$ at $4^{\circ} \mathrm{C}$. Supernatant was collected into a fresh microcentrifuge tube. One milliliter of base buffer was added to the pelleted cell lysate and syringe lysed again through a 22-gauge 1.5-inch needle 40 times. Lysates were centrifuged at $1,000 \mathrm{~g}$ for 10 $\min$ at $4^{\circ} \mathrm{C}$ and the second supernatant was combined with the first. One milliliter of base buffer was added to the pelleted cell lysate, syringe lysed again through a 22 -gauge 1.5 -inch needle 20 times and centrifuged at $1,000 \mathrm{~g}$ for $10 \mathrm{~min}$ at $4^{\circ} \mathrm{C}$. The third supernatant was combined with the previous two supernatants, resulting in approximately $3 \mathrm{ml}$ of cell lysate. One milliliter of OptiPrep density gradient medium (Sigma) was combined with the cell lysate at the bottom of a 12 -ml $9 / 16 \times 31 / 2^{\prime}$ open-top polyallomer centrifuge tube to comprise a $25 \%$ OptiPrep solution in base buffer (Denville Scientific, Metuchen, N.J., USA). A step gradient (22, 20, 18, 16, 14, 12, $10,8,6,4,2$ and $0 \%$ OptiPrep in base buffer) was carefully overlaid in $645-\mu \mathrm{l}$ increments on top of the $25 \%$ OptiPrep mixture. Centrifugation was then performed at $21,500 \mathrm{rpm}$ for $1.5 \mathrm{~h}$ at $4^{\circ} \mathrm{C}(\mathrm{SW}-41$ rotor; Beckman Coulter). After centrifugation, fractions were removed at $840-\mu \mathrm{l}$ increments from the top of the flotation gradient layer. Fractions were then subjected to immunoblot analysis.

\section{Infectivity Assays}

For serum starvation, FRhK4 cells in 24-well plates were incubated in $0.5 \%$ FBS-supplemented DMEM for a minimum of $6 \mathrm{~h}$ at $37^{\circ} \mathrm{C}$. Cell monolayers were washed once with PBS and then treated with $10 \mu \mathrm{M}$ C8-D-erythro-octanoyl-lactosylceramide (C8-D-eLacCer; Avanti Polar Lipids, Alabaster, Ala., USA) at room temperature for $15 \mathrm{~min}$. Cells were washed again with PBS and culture plates were incubated on ice with increasing volumes of HIV-1 or SIV GFP vectors for $30 \mathrm{~min}$ to synchronize particle binding. FRhK 4 cells were then incubated at $37^{\circ} \mathrm{C}$ for $10 \mathrm{~min}$ to facilitate particle entry into target cells. Immediately following the 10 -min incubation, cell monolayers were washed twice with PBS to remove free viral vector particles and replenished with 10\% FBSsupplemented DMEM. Approximately $36 \mathrm{~h}$ after infection, GFPexpressing cells were assayed by flow cytometry. Analysis was performed on Becton Dickinson CellQuest software (version 3.1; Becton Dickinson, Franklin Lakes, N.J., USA).

Fig. 1. The cytoplasmic body component TRIM5arh is rapidly recruited to thePMupon treatment with HIV-1 VLPs.aFRhK4T5aCh cells, which express an mCherry-labeled TRIM5arh protein, were incubated with or without HIV-1 VLPs expressing a GFP-labeled capsid on ice for $30 \mathrm{~min}$ and then immediately incubated at $37^{\circ} \mathrm{C}$ for 0,10 and 30 min to allow for particle fusion and entry. Cells were imaged in epifluorescence (Epi) and TIRF modes with an Olympus IX70 microscope equipped with $\times 601.4$ NA or $\times 1001.35$ NA oil immersion objectives. Images were acquired using a QuantEM:512SC (Photometrics) CCD camera. b The total number of pixel counts per cell detected in TIRF mode was quantified
Separation of Cytoplasmic and PM Fractions in T Cells

The Minute Plasma Membrane Isolation kit was used to isolate cytoplasmic and PM fractions from T cells (Invent Biotechnologies, Eden Prairie, Minn., USA). All procedures were performed on ice and followed the manufacturer's recommended protocols. HIV-1 VLP and C8-D-e-LacCer pretreatments were performed as described above.

\section{Immunoblotting}

Proteins were subjected to SDS-PAGE in $4-15 \%$ Tris- $\mathrm{HCl}$ gel and then transferred onto a PVDF membrane at $0.7 \mathrm{~mA} / \mathrm{cm}^{2}$ for 40 min. Membranes were blocked in 5\% milk in PBS overnight prior to application of antibodies. Antibodies were used in the following concentrations: mouse anti-flotillin-1/reggie-2 (1:1,000; BD Transduction Laboratories), mouse anti-flotillin-2/reggie-1 (1: 1,000; BD Transduction Laboratories), rat anti-HA (1:1,000; Roche), rabbit anti-TRIM5 (1:200; ProSci), mouse anti-p24 monoclonal antibodies, AG3.0 [45] and 183-H12-5C [46], and rabbit anti-Nup98 (1:500, a generous gift from Dr. Jan van Deursen). Peroxidase-conjugated secondary antibodies (goat anti-rat IgG, goat anti-mouse IgG and goat anti-rabbit IgG; Thermo Scientific, Waltham, Mass., USA) were used at a 1:2,000 concentration.

\section{Results}

\section{HIV-1 Particles Recruit TRIM5 $\alpha$ rh to the PM}

We first examined whether the cytoplasmic body component TRIM5arh could be visually detected at the host cell PM. FRhK4 cells stably expressing C-terminally mCherry-tagged TRIM5arh were subjected to analysis with TIRF microscopy, which can selectively illuminate and excite fluorophores within approximately $100 \mathrm{~nm}$ of the cell surface [47]. To synchronize HIV-1 cell surface binding, cells were incubated on ice for $30 \mathrm{~min}$ in the presence or absence of vesicular stomatitis virus (VSV)G-pseudotyped HIV-1 VLPs and further incubated at $37^{\circ} \mathrm{C}$ for 0,10 and $30 \mathrm{~min}$. When untreated cells were examined, epifluorescence imaging identified prominent TRIM5arh-mCherry cytoplasmic bodies, whereas TRIM5 arh signals at the PM were largely absent in TIRF mode (fig. 1a, no VLP control). Pre-incubation on ice did

using the MetaMorph image processing program (version 7.3.2; Universal Imaging); bars represent averages of 10-15 cells, error bars represent 1 SD and p was calculated using Student's t test. c Colocalization between signals from HIV-CA (green) and TRIM5arh (red) were observed $10 \mathrm{~min}$ after binding (colors refer to the online version only). d FRhK4T5aCh cells were incubated with SIV VLPs with a VSV-G pseudotype (SIV), BSA-coated nanoparticles [43] or HIV-1 particles without envelope pseudotype (Env-less VLPs) on ice for $30 \mathrm{~min}$ and then immediately incubated at $37^{\circ} \mathrm{C}$ for 10 min to allow for particle fusion and entry. Representative Epi and TIRF images are shown. 
a
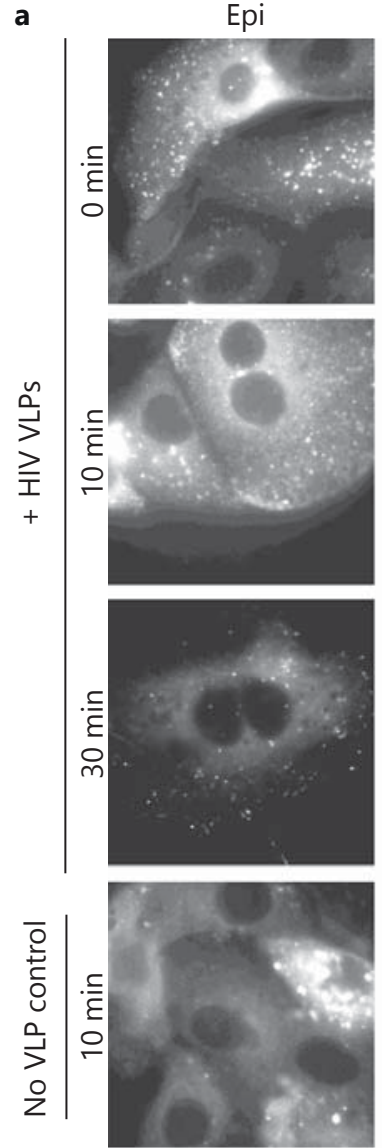

b

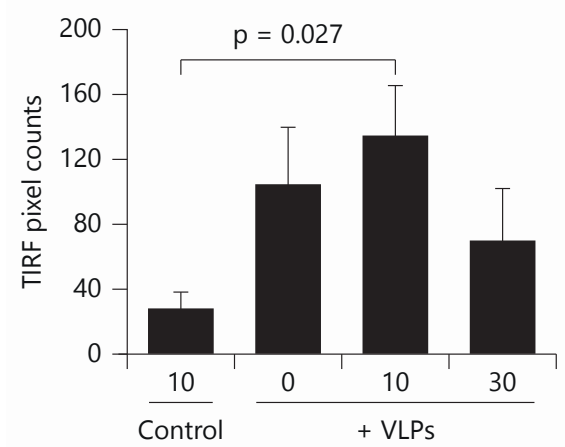

Time post-binding (min)

TIRF
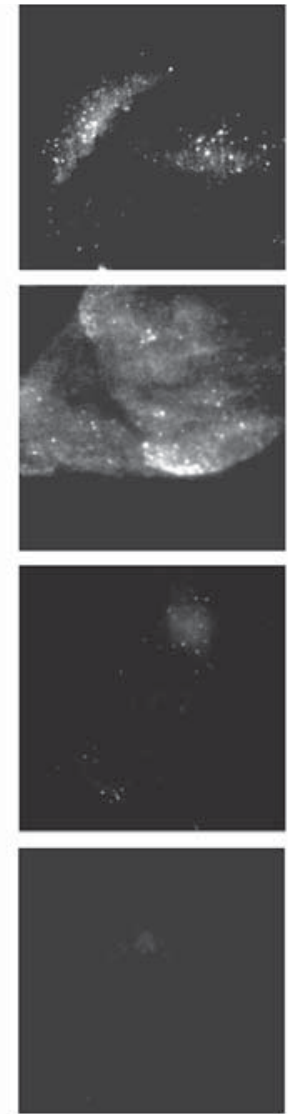

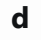

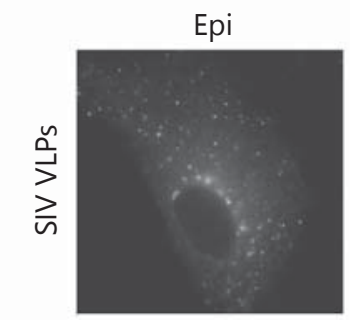
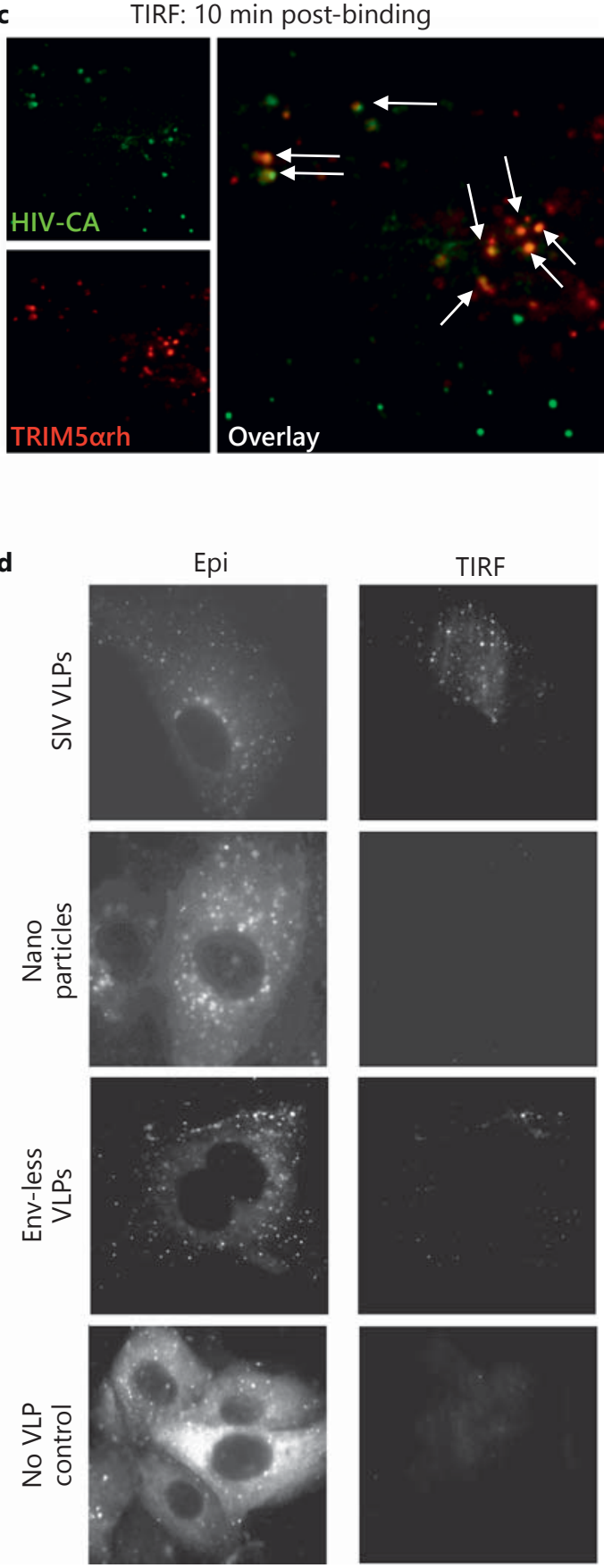

TIRF
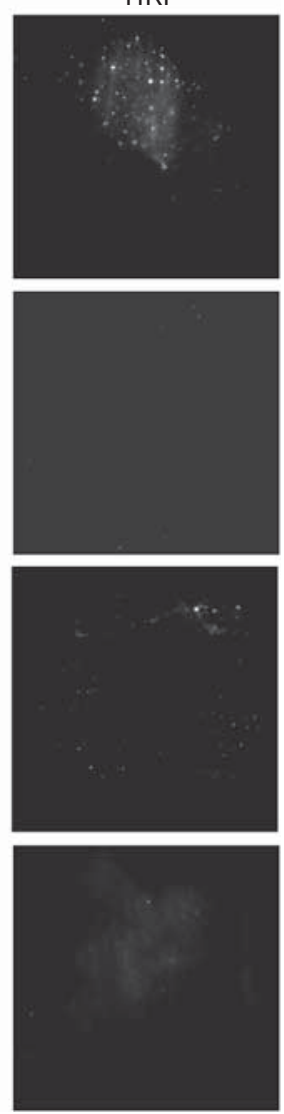
not affect the subcellular localization of TRIM5arh signals (data not shown). Those data demonstrated cytoplasmic localization of TRIM5arh in uninfected cells. When cells were incubated with the VLPs on ice, some TRIM5arh signals were recognized at the PM (fig. 1a, TIRF, $0 \mathrm{~min}$ ). Extended incubations at $37^{\circ} \mathrm{C}$ of $10 \mathrm{~min}$ led to a notable increase in the PM-detectable TRIM5arh signals in TIRF mode (fig. 1a). Quantification of TIRF signals showed significant increases in TRIM5arh signals at the PM upon incubation with the VLPs. TIRF signals were highest after the $10-\min 37^{\circ} \mathrm{C}$ incubation and decreased upon extended incubations of $30 \mathrm{~min}$ (fig. 1b). We speculate that rapid internalization of the proteinvirus complex may partially explain the observed decrease in TRIM5arh TIRF signals following the 30-min $37^{\circ} \mathrm{C}$ incubation (fig. 1a, b). Of note, when the TIRF signals from the HIV-1 CA-GFP particles and the TRIM5arh mCherry proteins were overlaid, we found frequent colocalization between the two signals at the PM (fig. 1c).

To determine the specificity of this TRIM5arh recruitment, we used VSV-G-pseudotyped VLPs made with TRIM5arh-resistant SIV, BSA-coated nanoparticles and HIV-1 VLPs devoid of the VSV-G Env (no-Env VLPs). Increased TRIM5 arh TIRF signals were detected in the presence of SIV particles after a 10 -min incubation at $37^{\circ} \mathrm{C}$ (fig. 1d), suggesting that the TRIM5arh PM recruitment occurs irrespective of the viral core restriction sensitivity. In contrast, the BSA-coated nanoparticles, which are known to rapidly internalize via albumin-mediated endocytosis [43], and the no-Env HIV-1 VLPs did not noticeably recruit TRIM5arh signals to the PM. Together, these data demonstrated that TRIM5arh is recruited to the PM immediately following VSV-G-pseudotyped VLP binding, but not upon albumin-mediated nanoparticle endocytosis.

\section{A Subpopulation of TRIM5arh Is Found in Higher \\ Buoyant Density Fractions}

We next examined for the presence of TRIM5arh in higher buoyant density (membrane lipid microdomain) fractions upon HIV-1 infection. We separated membrane raft fractions from whole cell lysates of FRhK4 cells stably expressing HA-tagged TRIM5arh protein (FRhK4T5a). To minimize the artifacts due to detergent-mediated protein aggregation, we used a detergent-free method [44]. As a marker for the flotation fractions, the membrane raft-associated protein flotillin-1 was assessed and nucleoporin Nup98 was used as a non-raft-associated control (fig. 2a). TRIM5arh signals were not detected higher than fraction 8 in untreated FRhK4T5 a cells; however, when cells were pretreated with HIV-1 VLPs, a separate
TRIM5arh signal was observed in the upper fraction of 4 and 5 (fig. 2a). p24 signals from the HIV-1 capsid were also detected in higher flotation fractions (fig. 2a). Enhanced flotillin- 1 signals in fractions 4 and 5 correspond to this TRIM5arh subpopulation found in higher buoyant density fractions.

In accordance with our TIRF analysis, treatment with the VSV-G-pseudotyped SIV VLPs also resulted in additional TRIM5arh-HA signals in fractions as high as 4 and 5 (fig. 2b), indicating that the recruitment of TRIM $5 \mathrm{arh}$ to the PM was independent of viral capsid identity. Since VSV-G pseudotyping mediates viral entry through endocytosis $[48,49]$, we examined whether TRIM5 arh recruitment has any involvement in the host cell endocytosis mechanisms. Upon treating FRhK4T5 a cells with BSAcoated nanoparticles, TRIM5arh signals were not apparent in the higher buoyant density fractions (fig. 2b).

To rule out the possibilities that the recruitment of TRIM5 arh is due to the exogenous expression of mCherry- or HA-tagged TRIM5arh proteins, we detected endogenous TRIM5arh using a commercially available antibody against TRIM5a (catalogue No. 3251; ProSci). Upon separation of higher buoyant density fractions, we were able to detect a specific band approximately $50 \mathrm{kDa}$ in size. Although only faint TRIM5arh bands were found in fractions 3-8, more prominent signals in these fractions were observed upon HIV-1 VLP binding to the PM under identical exposure conditions. These data suggest that a subset of endogenous TRIM5arh resides on the PM without viral exposure and that endogenous TRIM5arh is further recruited to the PM upon HIV-1 infection.

\section{A Glycosphingolipid Stereoisomer Can Recruit \\ TRIM5 $\alpha$ rh to the PM}

Brief incubation with exogenous glycosphingolipids stimulates the coalescence of membrane raft markers (such as $\beta_{1}$-integrin and $\mathrm{GM}_{1}$ ganglioside) into micron size structures at the PM $[50,51]$. Our previous proteomic studies have indicated that human TRIM5 $\alpha$ was more readily detected in higher buoyant density fractions upon aggregating PM lipids of human fibroblasts [36]. When FRhK4T5 $a$ cells were pretreated with $10 \mu \mathrm{M}$ C8-D-e-LacCer prior to detergent-free fractionation, an increased amount of TRIM $5 a-H A$ signal was detected in fraction 7 , as well as fainter signals in upper flotation fractions 2-6 (fig. 3a). Furthermore, TIRF analysis confirmed that C8D-e-LacCer treatment can recruit TRIM5arh to the host cell PM (fig. 3b). In previous reports, TRIM5a movement within the cytoplasm has been reported to be microtubule dependent [52]. We reasoned that this rapid TRIM5 $\alpha$ re- 
a

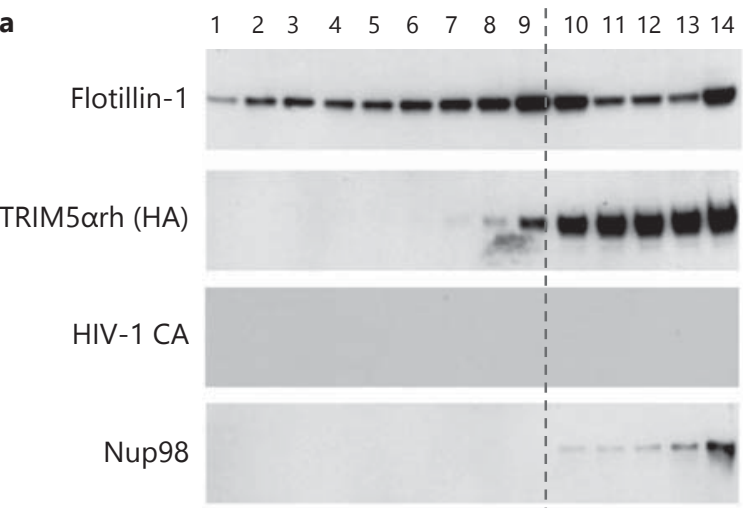

SIV

b

Flotillin-1

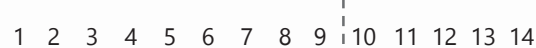

TRIM5 $\alpha$ rh (HA)
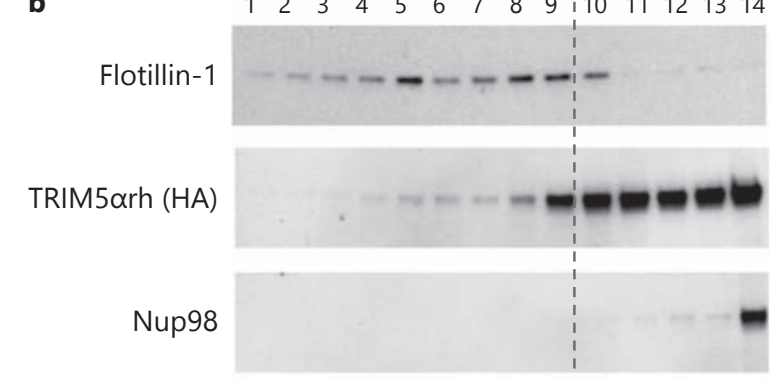

Control

c

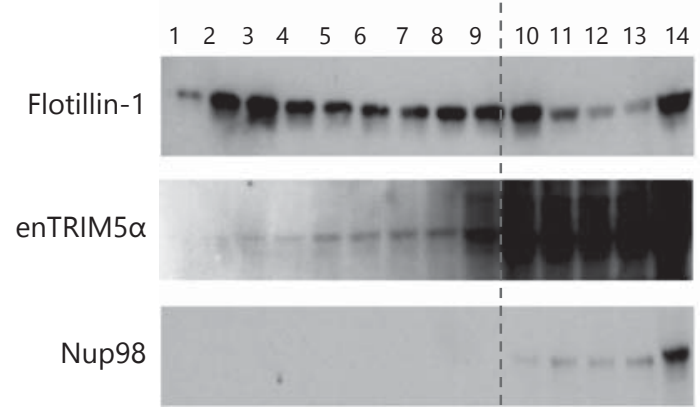

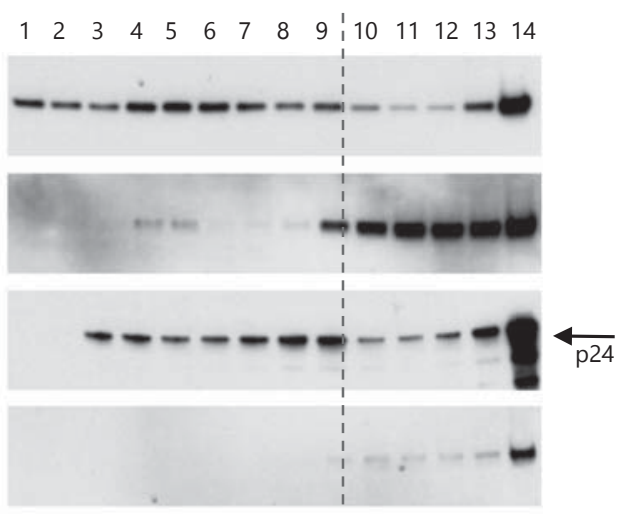

Nanoparticles

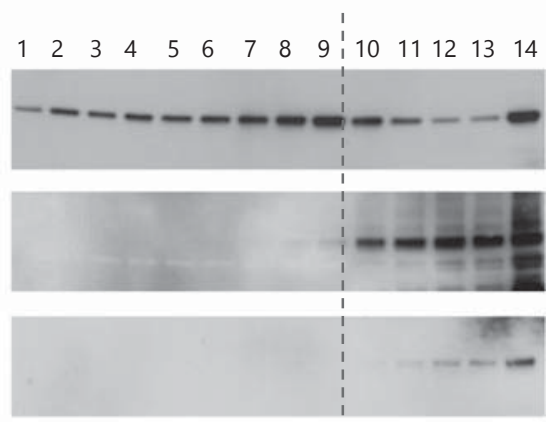

HIV-1

$\begin{array}{lllllllll:lllll}1 & 2 & 3 & 4 & 5 & 6 & 7 & 8 & 9 & 10 & 11 & 12 & 13 & 14\end{array}$
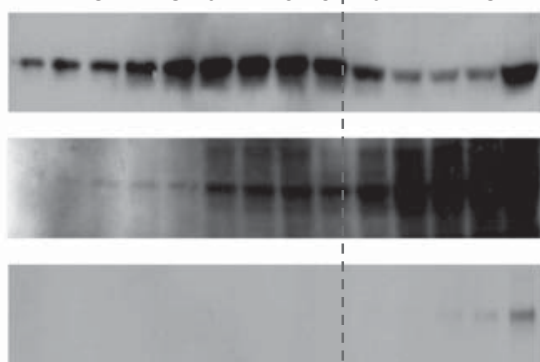

Fig. 2. TRIM5arh is found in higher buoyant density fractions upon VLP infection. A detergent-free method to separate higher buoyant density fractions was applied for all fractionation studies [44]. Representative images from 3 independent experiments are shown. a FRhK4 cells stably expressing HA-tagged TRIM5arh protein (FRhK4T5a-HA) treated with PBS (upper panel) or HIV-1 VLPs (lower panel) are shown. Flotillin-1 was used as a marker for flotation fractions, while Nup98 was used as a sedimenting control. Antibody against HIV-1 capsid (CA) is shown; p24 is indicated by arrow. b Identical experiments were carried out as in a, however using SIV VLPs or BSA-coated nanoparticles. c Identical fractionation experiments were carried out as in a, however using wild-type FRhK4 proteins expressing endogenous levels of TRIM5 arh (enTRIM $5 \mathrm{arh}$ ), which was probed using a commercially available antibody against TRIM5 (ProSci). 

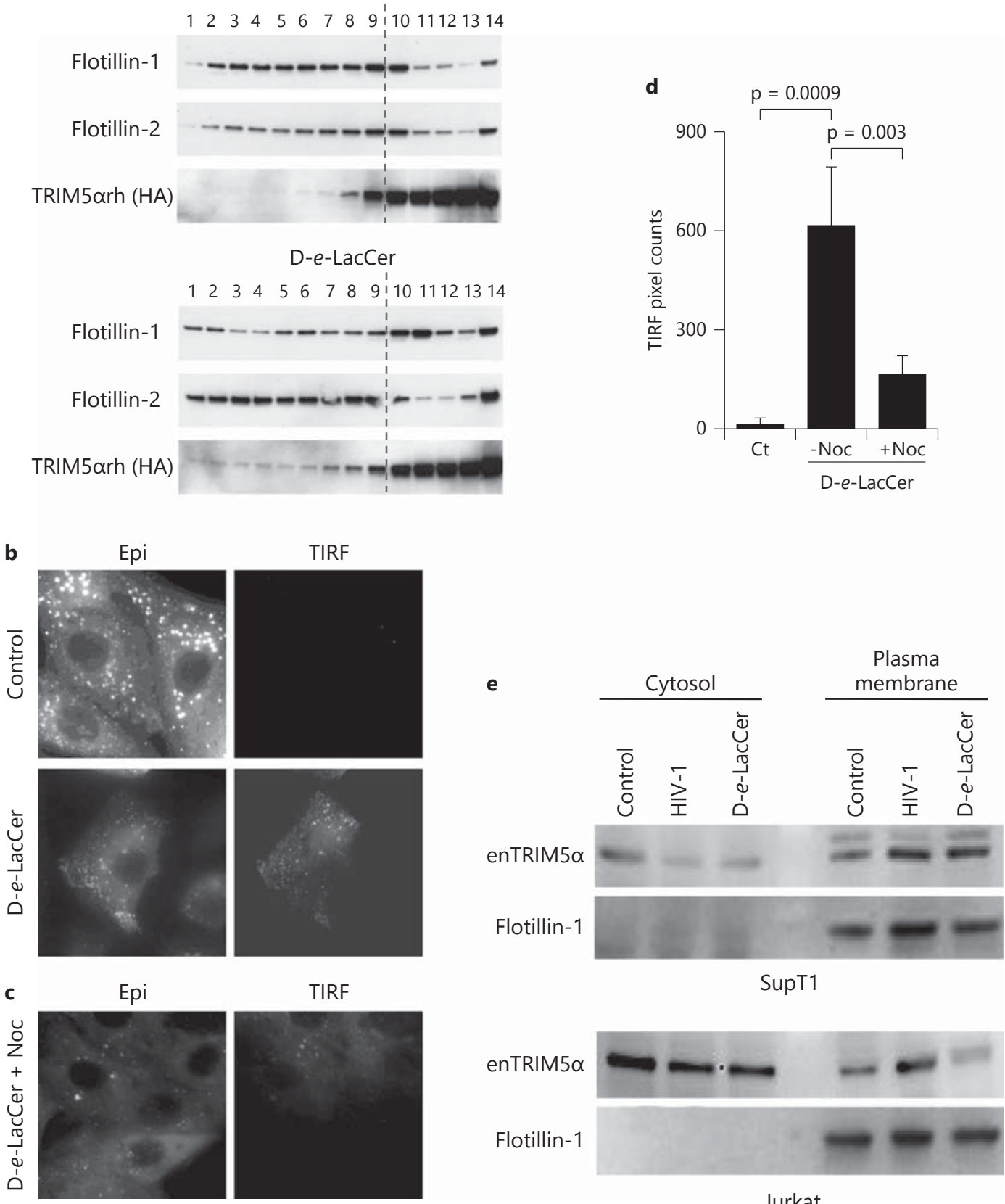

TIRF

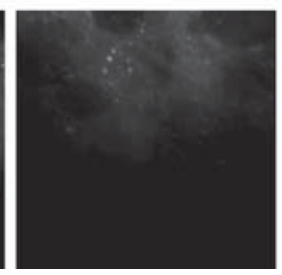

enTRIM $5 \alpha$

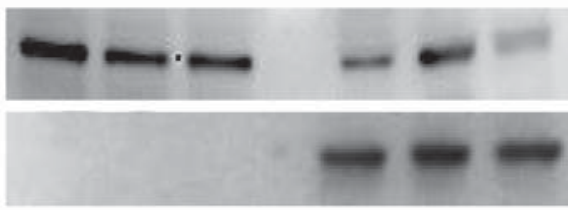

Jurkat

Fig. 3. Aggregation of PM lipids through glycosphingolipid stereoisomer incorporation also recruits TRIM5 arh to the PM. a Detergent-free isolation of higher buoyant density fractions of FRhK4 cells stably expressing HA-tagged TRIM5arh protein (FRhK4T5aHA) treated with vehicle control (left panel) or $10 \mu \mathrm{M}$ C8-D-e-LacCer (right panel) are shown (representative images from 3 independent experiments). b, c Epifluorescence (Epi) and TIRF images of FRhK4T $5 a \mathrm{Ch}$ cells which were serum starved, treated with vehicle control, or with $10 \mu \mathrm{M} \mathrm{C} 8$-D-e-LacCer for $15 \mathrm{~min}$ at room temperature or with or without a 2-hour pretreatment with $66 \mu \mathrm{M}$ nocodazole (Noc) to disrupt microtubule function (c). d Total number of pixels detected in TIRF mode were quantified using the MetaMorph image processing program (error bars represent $1 \mathrm{SD}$ and $\mathrm{p}$ values were calculated using Student's t test). $\mathrm{Ct}=$ Control. e Immunoblot analysis of SupT1 and Jurkat protein expression in the cytosol and PM following mock treatment (Control), HIV-1 Env-pseudotyped VLP binding (HIV-1) or treatment with $10 \mu \mathrm{M}$ C8-D-e-LacCer. TRIM5 and flotillin-1 proteins were detected using the above-described respective antibodies. enTRIM5arh = Endogenous levels of TRIM5arh. 


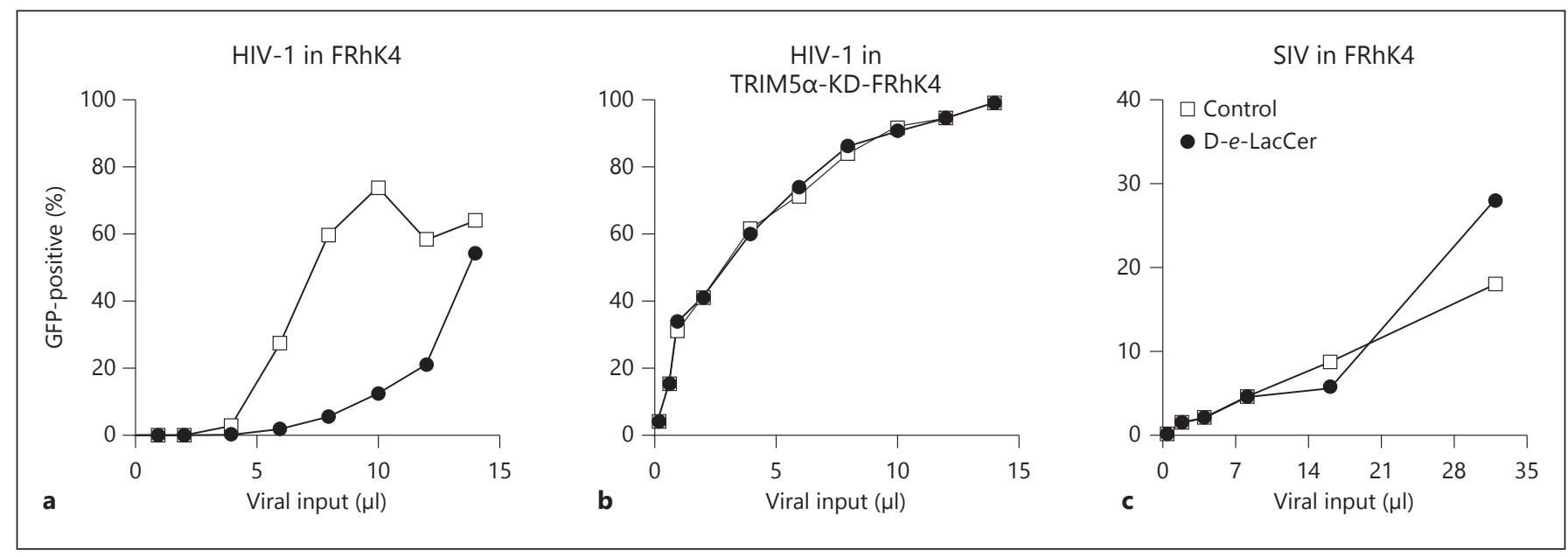

Fig. 4. Enhancement of TRIM5arh-mediated postentry restriction through PM prerecruitment. a FRhK4 cells were serum starved for $16 \mathrm{~h}$, treated with $10 \mu \mathrm{M}$ C8-D-e-LacCer for $15 \mathrm{~min}$ at room temperature and then infected on ice with increasing amounts of GFPexpressing HIV-1 vector particles. Virus particles were allowed to bind to the target cell PM for 30 min on ice and immediately incubated at $37^{\circ} \mathrm{C}$ for $10 \mathrm{~min}$. Excess particles were washed off with PBS, and media were replenished to the cells. GFP-positive cells were quantified using flow cytometry $48-72 \mathrm{~h}$ after infection. b FRhK4 cells knocked down for endogenous TRIM5arh expression were subjected to identical conditions as described in a. c FRhK4 cells were subjected to identical conditions as described in $\mathbf{a}$, however infected with GFP-expressing SIV vector particles, not HIV-1. Data are representative of at least 3 independent experiments. cruitment to the PM may also be dependent on microtubules. Indeed, when FRhK4T5 $a$ Ch cells were treated with $66 \mu \mathrm{M}$ nocodazole for $2 \mathrm{~h}$ prior to $10-\mu \mathrm{M} C 8-\mathrm{D}-e$-LacCer treatment, a remarkable decrease in TRIM5 $\alpha$ signals at the PM was observed (fig. 3c, d).

Next, we determined the possible PM recruitment of endogenous human TRIM5 $a$ in T lymphocytes. SupT1 and Jurkat cells were pretreated with HIV-1 Env-pseudotyped HIV-1 VLPs or C8-D-e-LacCer, then subjected to $\mathrm{PM}$ isolation by a commercially available PM isolation kit, which facilitates separation of cytoplasmic and PM protein fractions (Invent Biotechnologies). Upon immunoblot analysis of each cytoplasmic and PM fraction, endogenous TRIM5 $a$ signals were higher in the PM fractions upon pretreatment with HIV-1 VLPs and, to a lesser degree, with C8-D-e-LacCer (fig. 3e). These data suggest that endogenous human TRIM5 $\alpha$ in T cells can be recruited to the PM upon exposure to HIV-1 VLPs.

\section{Prerecruitment of TRIM5 $\alpha$ rh to the PM Enhances the}

\section{Postentry Restriction}

We then examined the role of TRIM5arh PM recruitment on the restriction activity against incoming virus particles. We pretreated FRhK4 cells with $10 \mu \mathrm{M}$ of C8-D$e$-LacCer and then applied VSV-G-pseudotyped HIV-1 GFP vectors at increasing concentrations, which could saturate the endogenous postentry restriction. The virus par- ticles were incubated with the target cells for $30 \mathrm{~min}$ on ice and allowed for viral entry through a 10 -min incubation at $37^{\circ} \mathrm{C}$. When cells were pretreated with C8-D-e-LacCer, infection kinetics were changed, and cells were more resistant to HIV-1 GFP vector infection. The potency of restriction was enhanced approximately 5 -fold (fig. 4a), although the saturation of the restriction was eventually observed at higher viral doses. When endogenous TRIM5arh expression was stably knocked down, there was no change in HIV-1 infectivity with or without C8-D-e-LacCer pretreatment (fig. 4b). Similarly, C8-D-e-LacCer did not alter the infectivity of a permissive SIV GFP vector in FRhK4 cells (fig. 4c). These data demonstrate that the recruitment of TRIM5 arh to the PM plays an important role in HIV-1 capsid restriction. Our data also indicate that the C8-D-eLacCer-mediated enhanced postentry restriction is dependent on TRIM5arh expression and a TRIM5arhrestricted virus, and rule out the possible nonspecific C8$\mathrm{D}$-e-LacCer effects on viral infectivity.

\section{Discussion}

TRIM5 $\alpha$ is a multifunctional component of the innate immune system $[53,54]$. In addition to restricting retroviral infection, recognition of the hexameric capsid lattice, a molecular signature of retroviruses, also leads to 
activation of the TAK1 kinase complex and subsequent stimulation of AP-1 and NFKB signaling [23, 53, 54]. Here, we have demonstrated that retroviral attachment and PM microdomain clustering rapidly recruited TRIM5 $\alpha$ to lipid-enriched PM regions. Of note, prominent accumulations of PM TRIM5arh signals were observed at the sites of viral attachment/entry after a 10-min incubation at $37^{\circ} \mathrm{C}$. Although previous studies have demonstrated that cytoplasmic TRIM5 $\alpha$ bodies are highly mobile [52], our data indicate that the rapid PM recruitment of TRIM5 $\alpha$ is a preceding step for innate retroviral detection.

Efficient host cell restriction of an incoming virus particle requires rapid recognition of viral components. In the postentry HIV-1 restriction by TRIM5arh, recognition is efficient and rapid $[9,55]$. Thus, there either must be an abundance of the restriction factor in the cytoplasm or strategic availability of TRIM5 $\alpha$ proteins in areas with high viral traffic. Previous studies have demonstrated through the ease of abrogation that restriction factors are of limited availability $[12,56,57]$, thus supporting the latter hypothesis. Our study demonstrated the importance of PM TRIM5 $\alpha$ recruitment for efficient HIV-1 restriction. Notably, when endogenous TRIM5arh in FRhK4 cells was detected by the flotation assays, only a marginal proportion of TRIM5arh was in higher buoyant density fractions. Exposure to HIV-1 VLPs strongly increased the levels of TRIM5arh in flotation fractions, although the majority of endogenous TRIM5arh remained in the lower buoyant densityfractions. Given thelimitedavailabilityofTRIM5 $\mathrm{arh}$ for restriction in FRhK4 cells, it is conceivable that the PMassociated higher buoyant density TRIM5arh plays a key role in retroviral restriction. We speculate that the lower buoyant density TRIM5 $a$ is involved in the ensuing retroviral restriction process, such as induction of proteasomal degradation of the retroviral capsid. Since the cytoplasmic colocalization between exogenously expressed TRIM5 a and target viral capsid has been reported $[35,52,58]$, it is also possible that the lower buoyant density TRIM5arh may play a secondary role, such as blocking incoming viruses in cytoplasm or promoting higher-order TRIM5arh self-association for enhanced viral restriction [59].

Our results demonstrated the rapid TRIM5 $a$ recruitment to the host cell PM upon viral particle binding. The rapid recruitment was observed when cells were incubated with VSV-G-pseudotyped HIV-1 and SIV VLPs (fig. 1b, d), but not by artificial BSA-coated nanoparticles (fig. 1d). Although no-Env HIV-1 VLPs failed to recruit TRIM5arh, the binding of HIV-1 Env-pseudotyped HIV1 VLPs could recruit TRIM5 $\alpha$ in human lymphocytes.
These observations indicate that (i) the recruitment occurs irrespectively of the viral core sensitivity to the TRIM5 $\alpha$; (ii) albumin-mediated endocytosis does not induce TRIM5 $\alpha$ recruitment, and (iii) both VSV-G and HIV-1-Env pseudotypes can induce this recruitment. Since initiation of the TRIM5a PM recruitment can be seen when cells were maintained on ice for $30 \mathrm{~min}$, we argue that TRIM5 $\alpha$ recruitment to the PM does not require viral entry into the cells. Interestingly, similar TRIM5 a PM recruitment was observed when PM microdomains were clustered using C8-D-e-LacCer (fig. 3b). Additionally, this recruitment was also dependent on intact microtubules (fig. 3c, d). Since no-Env VLPs failed to recruit TRIM5 $\alpha$, it is plausible that TRIM5 $\alpha$ PM recruitment is induced by the changes in membrane potential or a clustering of PM microdomains through viral receptor binding (such as CD4 and CCR5, which reside in regions enriched in steric lipids). Alternatively, binding of pathogen-associated molecular patterns, such as abnormal lipid contents in viral glycoproteins, may play a role in triggering the PM recruitment of TRIM5a.

Previously, we identified increased levels of endogenous human TRIM5 $a$ in PM lipid microdomains after short-term stimulation with a synthetic glycosphingolipid analog [36]. In this study, we also demonstrate the rapid PM recruitment of endogenous human TRIM5a upon infection with HIV-1 Env-pseudoypted HIV-1 vectors in human T lymphocytes (fig. 3e). Considering the primary receptor and coreceptors for HIV-1 are known to reside in membrane raft domains, endogenous TRIM5 $a$ recruitment to the lipid-enriched viral entry hot spots is likely to set up a formidable first-line antiviral defense. A recent report suggests that permissivity to HIV-1 infection is partially dependent on the TRIM5 $\alpha$ context [60], warranting further studies in human lymphocytes to determine this antiviral context of TRIM5a.

Human TRIM5 $a$ is known to restrict N-tropic, but not $\mathrm{B}$-tropic, murine leukemia virus (MLV). It remains to be determined whether MLV infection also recruits human TRIM5 $a$ to the PM. As mentioned above, our study demonstrates the promiscuous recruitment of TRIM $5 a$ to the PM by HIV-1 Env-pseudotyped HIV-1, VSV-G-pseudotyped HIV-1 and SIVmac, irrespective of their sensitivity to TRIM5a. Although further studies are needed, we speculate that exposure to VSV-G-pseudotyped N-MLV or B-MLV will lead to human TRIM5a recruitment.

In summary, our findings demonstrated the rapid translocation of TRIM5 $a$ to the host cell PM upon exposure to HIV-1 VLPs and PM lipid clustering. Recruitment of TRIM5arh to the PM, prior to viral exposure, increased 
the potency of postentry restriction activity against incoming HIV-1 particles. Our data therefore suggest that the rapid PM recruitment of TRIM5a facilitates efficient innate immune sensing and restriction of retroviral infection.

\section{Acknowledgments}

We would like to acknowledge Dr. Richard E. Pagano, who passed away in late 2010 after an extended illness. This work would not have been possible without his guidance and expertise. The following reagents were obtained through the NIH AIDS Research and Reference Reagent Program (Division of AIDS, NIAD, NIH): HIV-1 p24 monoclonal antibodies 183-H12-5C from Dr. B. Chesebro and K. Wehrly, AG3.0 from Dr. J. Allan and pDOLHIVenv from Dr. Eric Freed and Dr. Rex Risser. This work was supported by NIH 1R56AI074363-01A1 (to Y. I.), NIH R01GM22942 (to R.E.P.) and the Mayo Foundation (to Y. I.).

\section{Disclosure Statement}

The authors have no competing interests to disclose.

\section{References}

1 Perron MJ, Stremlau M, Song B, Ulm W, Mulligan RC, Sodroski J: TRIM5alpha mediates the postentry block to $\mathrm{N}$-tropic murine leukemia viruses in human cells. Proc Natl Acad Sci USA 2004;101:11827-11832.

$\checkmark 2$ Hatziioannou T, Perez-Caballero D, Yang A, Cowan S, Bieniasz PD: Retrovirus resistance factors Ref1 and Lv1 are species-specific variants of TRIM5alpha. Proc Natl Acad Sci USA 2004;101:10774-10779.

3 Yap MW, Nisole S, Lynch C, Stoye JP: Trim5alpha protein restricts both HIV-1 and murine leukemia virus. Proc Natl Acad Sci USA 2004;101:10786-10791.

-4 Stremlau M, Owens CM, Perron MJ, Kiessling M, Autissier P, Sodroski J: The cytoplasmic body component TRIM5alpha restricts HIV1 infection in Old World monkeys. Nature 2004;427:848-853.

5 Nisole S, Stoye JP, Saib A: TRIM family proteins: retroviral restriction and antiviral defence. Nat Rev Microbiol 2005;3:799-808.

6 Ozato K, Shin DM, Chang TH, Morse HC 3rd: TRIM family proteins and their emerging roles in innate immunity. Nat Rev Immunol 2008;8:849-860.

7 Perez-Caballero D, Hatziioannou T, Yang A, Cowan S, Bieniasz PD: Human tripartite motif $5 a$ domains responsible for retrovirus restriction activity and specificity. J Virol 2005; 79:8969-8978.

8 Song B, Javanbakht H, Perron M, Park DH, Stremlau M, Sodroski J: Retrovirus restriction by TRIM5alpha variants from Old World and New World primates. J Virol 2005;79:39303937.

-9 Stremlau M, Perron M, Lee M, Li Y, Song B, Javanbakht H, Diaz-Griffero F, Anderson DJ, Sundquist WI, Sodroski J: Specific recognition and accelerated uncoating of retroviral capsids by the TRIM5 $\alpha$ restriction factor. Proc Natl Acad Sci USA 2006;103:5514-5519.

10 Yap MW, Nisole S, Stoye JP: A single amino acid change in the SPRY domain of human Trim5alpha leads to HIV-1 restriction. Curr Biol 2005;15:73-78.
11 Cowan S, Hatziioannou T, Cunningham T, Muesing MA, Gottlinger HG, Bieniasz PD: Cellular inhibitors with Fv1-like activity restrict human and simian immunodeficiency virus tropism. Proc Natl Acad Sci USA 2002; 99:11914-11919.

12 Kootstra NA, Munk C, Tonnu N, Landau NR, Verma IM: Abrogation of postentry restriction of HIV-1-based lentiviral vector transduction in simian cells. Proc Natl Acad Sci USA 2003;100:1298-1303.

13 Münk C, Brant SM, Lucero G, Landau NR: A dominant block to HIV-1 replication at reverse transcription in simian cells. Proc Natl Acad Sci USA 2002;99:13843-13848.

14 Owens CM, Song B, Perron MJ, Yang PC, Stremlau M, Sodroski J: Binding and susceptibility to postentry restriction factors in monkey cells are specified by distinct regions of the human immunodeficiency virus type 1 capsid. J Virol 2004;78:5423-5437.

15 Ganser-Pornillos BK, Chandrasekaran V, Pornillos O, Sodroski JG, Sundquist WI, Yeager M: Hexagonal assembly of a restricting TRIM5alpha protein. Proc Natl Acad Sci USA 2011;108:534-539.

16 Keckesova Z, Ylinen LM, Towers GJ: The human and African green monkey TRIM5alpha genes encode Ref1 and Lv1 retroviral restriction factor activities. Proc Natl Acad Sci USA 2004;101:10780-10785.

17 Chatterji U, Bobardt MD, Gaskill P, Sheeter D, Fox H, Gallay PA: Trim5alpha accelerates degradation of cytosolic capsid associated with productive HIV-1 entry. J Biol Chem 2006;281:37025-37033.

18 Akira S, Uematsu S, Takeuchi O: Pathogen recognition and innate immunity. Cell 2006; 124:783-801.

19 Wilkins C, Gale M Jr: Recognition of viruses by cytoplasmic sensors. Curr Opin Immunol 2010;22:41-47.
20 Kang DC, Gopalkrishnan RV,Wu Q, Jankowsky E, Pyle AM, Fisher PB: mda-5: an interferoninducible putative RNA helicase with doublestranded RNA-dependent ATPase activity and melanoma growth-suppressive properties. Proc Natl Acad Sci USA 2002;99:637-642.

21 Kato H, Takeuchi O, Sato S, Yoneyama M, Yamamoto M, Matsui K, Uematsu S, Jung A, Kawai T, Ishii KJ, Yamaguchi O, Otsu K, Tsujimura T, Koh CS, Reis e Sousa C, Matsuura Y, Fujita T, Akira S: Differential roles of MDA5 and RIG-I helicases in the recognition of RNA viruses. Nature 2006;441:101-105.

22 Gitlin L, Barchet W, Gilfillan S, Cella M, Beutler B, Flavell RA, Diamond MS, Colonna M: Essential role of mda- 5 in type I IFN responses to polyriboinosinic: polyribocytidylic acid and encephalomyocarditis picornavirus. Proc Natl Acad Sci USA 2006;103:8459-8464.

-23 Pertel T, Hausmann S, Morger D, Zuger S, Guerra J, Lascano J, Reinhard C, Santoni FA, Uchil PD, Chatel L, Bisiaux A, Albert ML, Strambio-De-Castillia C, Mothes W, Pizzato M, Grutter MG, Luban J: TRIM5 is an innate immune sensor for the retrovirus capsid lattice. Nature 2011;472:361-365.

24 Simons K, Ikonen E: Functional rafts in cell membranes. Nature 1997;387:569-572.

25 Guyader M, Kiyokawa E, Abrami L, Turelli P, Trono D: Role for human immunodeficiency virus type 1 membrane cholesterol in viral internalization. J Virol 2002;76:10356-10364.

26 Nguyen DH, Taub D: CXCR4 function requires membrane cholesterol: implications for HIV infection. J Immunol 2002;168:4121-4126.

27 Popik W, Alce TM, Au WC: Human immunodeficiency virus type 1 uses lipid raft-colocalized CD4 and chemokine receptors for productive entry into CD4(+) T cells. J Virol 2002;76:4709-4722.

28 Manes S, del Real G, Lacalle RA, Lucas P, Gomez-Mouton C, Sanchez-Palomino S, Delgado R, Alcami J, Mira E, Martinez AC: Membrane raft microdomains mediate lateral assemblies required for HIV-1 infection. EMBO Rep 2000;1:190-196.
TRIM5a Recruitment to the Plasma Membrane 
-29 Ablan S, Rawat SS, Viard M, Wang JM, Puri A, Blumenthal R: The role of cholesterol and sphingolipids in chemokine receptor function and HIV-1 envelope glycoprotein-mediated fusion. Virol J 2006;3:104.

- 30 Singer II, Scott S, Kawka DW, Chin J, Daugherty BL, DeMartino JA, DiSalvo J, Gould SL, Lineberger JE, Malkowitz L, Miller MD, Mitnaul L, Siciliano SJ, Staruch MJ, Williams HR, Zweerink HJ, Springer MS: CCR5, CXCR4, and CD4 are clustered and closely apposed on microvilli of human macrophages and T cells. J Virol 2001;75:3779-3790.

- 31 Carter GC, Bernstone L, Sangani D, Bee JW, Harder T, James W: HIV entry in macrophages is dependent on intact lipid rafts. Virology 2009;386:192-202.

- 32 del Real G, Jimenez-Baranda S, Lacalle RA, Mira E, Lucas P, Gomez-Mouton C, Carrera AC, Martinez AC, Manes S: Blocking of HIV1 infection by targeting CD4 to nonraft membrane domains. J Exp Med 2002;196:293-301.

-33 Kozak SL, Heard JM, Kabat D: Segregation of CD4 and CXCR4 into distinct lipid microdomains in $\mathrm{T}$ lymphocytes suggests a mechanism for membrane destabilization by human immunodeficiency virus. J Virol 2002;76: 1802-1815.

- 34 Hwang CY, Holl J, Rajan D, Lee Y, Kim S, Um M, Kwon KS, Song B: Hsp70 interacts with the retroviral restriction factor TRIM5alpha and assists the folding of TRIM5alpha. J Biol Chem 2010;285:7827-7837.

- 35 Ohmine S, Sakuma R, Sakuma T, Thatava T, Solis GP, Ikeda Y: Cytoplasmic body component TRIM5 $a$ requires lipid-enriched microdomains for efficient HIV-1 restriction. J Biol Chem 2011;285:34508-34517.

- 36 Kim SY, Wang TK, Singh RD, Wheatley CL, Marks DL, Pagano RE: Proteomic identification of proteins translocated to membrane microdomains upon treatment of fibroblasts with the glycosphingolipid, C8-beta-D-lactosylceramide. Proteomics 2009;9:4321-4328.

-37 Sakuma R, Noser JA, Ohmine S, Ikeda Y: Rhesus monkey TRIM5alpha restricts HIV-1 production through rapid degradation of viral Gag polyproteins. Nat Med 2007;13:631-635.

-38 Zufferey R, Nagy D, Mandel RJ, Naldini L, Trono D: Multiply attenuated lentiviral vector achieves efficient gene delivery in vivo. Nat Biotechnol 1997;15:871-875.
39 Noser JA, Towers GJ, Sakuma R, Dumont JM, Collins MK, Ikeda Y: Cyclosporine increases human immunodeficiency virus type 1 vector transduction of primary mouse cells. J Virol 2006;80:7769-7774.

40 Sakuma R, Mael AA, Ikeda Y: Alpha interferon enhances TRIM5alpha-mediated antiviral activities in human and rhesus monkey cells. J Virol 2007;81:10201-10206.

41 Freed EO, Myers DJ, Risser R: Mutational analysis of the cleavage sequence of the human immunodeficiency virus type 1 envelope glycoprotein precursor gp160. J Virol 1989; 63:4670-4675.

42 Negre D, Mangeot PE, Duisit G, Blanchard S, Vidalain PO, Leissner P, Winter AJ, Rabourdin-Combe C, Mehtali M, Moullier P, Darlix JL, Cosset FL: Characterization of novel safe lentiviral vectors derived from simian immunodeficiency virus (SIVmac251) that efficiently transduce mature human dendritic cells. Gene Ther 2000;7:1613-1623.

43 Wang Z, Tiruppathi C, Minshall RD, Malik $\mathrm{AB}$ : Size and dynamics of caveolae studied using nanoparticles in living endothelial cells. ACS Nano 2009;3:4110-4116.

44 Macdonald JL, Pike LJ: A simplified method for the preparation of detergent-free lipid rafts. J Lipid Res 2005;46:1061-1067.

45 Simm M, Shahabuddin M, Chao W, Allan JS, Volsky DJ: Aberrant Gag protein composition of a human immunodeficiency virus type 1 vif mutant produced in primary lymphocytes. J Virol 1995;69:4582-4586.

46 Chesebro B, Wehrly K, Nishio J, Perryman S: Macrophage-tropic human immunodeficiency virus isolates from different patients exhibit unusual $v 3$ envelope sequence homogeneity in comparison with T-cell-tropic isolates: definition of critical amino acids involved in cell tropism. J Virol 1992;66:6547-6554.

47 Axelrod D: Cell-substrate contacts illuminated by total internal reflection fluorescence. J Cell Biol 1981;89:141-145.

48 Aiken C: Pseudotyping human immunodeficiency virus type 1 (HIV-1) by the glycoprotein of vesicular stomatitis virus targets HIV1 entry to an endocytic pathway and suppresses both the requirement for Nef and the sensitivity to cyclosporin A. J Virol 1997;71: 5871-5877.
49 Luo T, Douglas JL, Livingston RL, Garcia JV: Infectivity enhancement by HIV-1 Nef is dependent on the pathway of virus entry: implications for HIV-based gene transfer systems. Virology 1998;241:224-233.

50 Sharma DK, Brown JC, Cheng Z, Holicky EL, Marks DL, Pagano RE: The glycosphingolipid, lactosylceramide, regulates betal-integrin clustering and endocytosis. Cancer Res 2005; 65:8233-8241.

51 Singh RD, Holicky EL, Cheng ZJ, Kim SY, Wheatley CL, Marks DL, Bittman R, Pagano RE: Inhibition of caveolar uptake, SV40 infection, and betal-integrin signaling by a nonnatural glycosphingolipid stereoisomer. J Cell Biol 2007;176:895-901.

52 Campbell EM, Dodding MP, Yap MW, Wu X, Gallois-Montbrun S, Malim MH, Stoye JP, Hope TJ: TRIM5 alpha cytoplasmic bodies are highly dynamic structures. Mol Biol Cell 2007;18:2102-2111.

53 Luban J: TRIM5 and the regulation of HIV-1 infectivity. Mol Biol Int 2012;2012:426840.

54 Nakayama EE, Shioda T: Role of human TRIM5alpha in intrinsic immunity. Front Microbiol 2012;3:97.

55 Rold CJ, Aiken C: Proteasomal degradation of TRIM5alpha during retrovirus restriction. PLoS Pathog 2008;4:e1000074.

56 Besnier C, Takeuchi Y, Towers G: Restriction of lentivirus in monkeys. Proc Natl Acad Sci USA 2002;99:11920-11925.

57 Shi J, Aiken C: Saturation of TRIM5 alphamediated restriction of HIV-1 infection depends on the stability of the incoming viral capsid. Virology 2006;350:493-500.

-58 Campbell EM, Perez O, Anderson JL, Hope TJ: Visualization of a proteasome-independent intermediate during restriction of HIV-1 by rhesus TRIM5alpha. J Cell Biol 2008;180: 549-561.

59 Diaz-Griffero F, Qin XR, Hayashi F, Kigawa T, Finzi A, Sarnak Z, Lienlaf M, Yokoyama S, Sodroski J: A B-box 2 surface patch important for TRIM5alpha self-association, capsid binding avidity, and retrovirus restriction. J Virol 2009;83:10737-10751

60 Battivelli E, Migraine J, Lecossier D, Yeni P, Clavel F, Hance AJ: Gag cytotoxic T lymphocyte escape mutations can increase sensitivity of HIV-1 to human TRIM5alpha, linking intrinsic and acquired immunity. J Virol 2012; 85:11846-11854. 